\title{
Coping, affective distress and psychosocial adjustment among people with traumatic upper limb amputations
}

\author{
Deirdre M Desmond PhD \\ Department of Psychology, John Hume Building, National University of Ireland \\ Maynooth, Maynooth, Co. Kildare, Ireland \\ and \\ Dublin Psychoprosthetics Group, Dublin, Ireland \\ http://www.tcd.ie/Psychoprosthetics/ \\ *Correspondence \& Requests for Reprints: \\ Dr. Deirdre Desmond, Department of Psychology, John Hume Building, National \\ University of Ireland, Maynooth, Maynooth, Co. Kildare, \\ E-mail: Deirdre.Desmond@ @uim.ie
} Telephone: 353-1-708 6479 Fax: 353-1-708 4767

Running head: Coping and adjustment to traumatic upper limb amputation 


\section{Acknowledgements}

This research was funded by the Irish Research Council for the Humanities and Social Sciences, and the British Limbless Ex-Service Men's Association (BLESMA). A debt of gratitude is owed to the individual members of BLESMA who took great time and care in responding to the survey, to Jerome Church and Stephen Coltman at BELSMA HQ for their assistance, to Professor Malcolm MacLachlan, School of Psychology, Trinity College Dublin, for his guidance and to three anonymous reviewers for their constructive comments. 


\begin{abstract}
Objective: This study investigated the prevalence of symptoms of depression and anxiety in a sample of predominantly elderly males with acquired upper limb amputations $(n=138)$ and examined the contribution of coping strategies to the prediction of psychosocial adjustment. Method: One-hundred and thirty-eight men with injury-related upper limb amputations completed self-report questionnaires assessing coping strategies, symptoms of anxiety and depression, and psychosocial adaptation to prosthesis use. Results: Prevalence of significant depressive symptoms was $28.3 \%$ (HADS-D score $\geq 8$ ). Prevalence of significant anxiety symptoms was $35.5 \%$ (HADS-A score $\geq 8$ ). Coping styles emerged as important predictors of psychosocial adaptation. In particular, avoidance was strongly associated with psychological distress and poor adjustment. Conclusions: These findings suggest the potential benefits of interventions to reduce reliance on avoidant coping and stimulate more problem-focused approaches to coping with difficulties and challenges in order to facilitate adaptation and prevent problems in psychosocial functioning postamputation.
\end{abstract}

Key words: Amputation; Coping; Psychosocial Adjustment; Upper limb; 


\section{Introduction}

Amputation of an upper limb engenders a multitude of physical and psychosocial challenges including alterations in body image and lifestyle, changes in self concept, impairments in physical functioning, prosthesis use and pain (1-3). The complexity and diversity of functions performed by the hands and their salience in communication and self-presentation (4) represent significant and distinct challenges for rehabilitation and prosthetic restoration. In recent years, significant technological advances in design and fabrication of upper limb prosthetic devices have greatly improved the potential functional and cosmetic outcomes for individuals with upper limb amputations (5). There remains, however, a dearth of research explicitly addressing psychosocial adaptation to upper limb amputation and associated mediating factors (6), despite the importance of such variables in clinical rehabilitation, sustained prosthesis use and long-term adjustment and quality of life.

It is not surprising that negotiating the evolving stressors associated with amputation may challenge the individuals' ability to maintain emotional well-being and, in some instances, may promote maladaptive reactions leading to poor psychosocial adjustment. The extent to which this occurs may be partly dependent on the coping strategies or styles individuals adopt to manage experiences associated with their illness or injury (7-10). Investigation of the role of coping strategies in adjustment to lower limb amputation, consistent with the wider coping literature, suggests that active/task oriented strategies such as problem solving and perceiving control over the disability are conducive to positive psychosocial adjustment (e.g. $7,11,12$ ). In contrast, emotion-focused and passive strategies such as cognitive disengagement, avoidance and catastrophizing have been associated with poor psychosocial outcomes 
$(7,12,13)$. For example, Livneh, Antonak \& Gerhard (7) found that greater active problem solving was negatively associated with depression and internalised anger and positively associated with adjustment and acceptance of disability. In contrast, emotion focused coping and cognitive disengagement were positively associated with depression, externalised hostility and lack of acceptance of disability.

Despite a large and growing literature on psychosocial adaptation to lower limb amputation (see 14 for review) there is little evidence regarding the prevalence of clinically significant affective distress amongst individuals with upper limb amputations. Indeed, to date associations between coping strategies and psychosocial adjustment to upper limb amputation, as a unique condition, have not been investigated. This may be explained in terms of the lower incidence of major upper limb amputation. However, findings based on analyses of cases of lower limb amputation are clearly of limited generalizeability to cases of upper limb amputation. There are obvious differences in terms of functional implications, visibility/concealability of the amputation and/or prosthesis, and in the characteristic circumstances surrounding upper and lower limb amputations. Amputations of the upper limbs typically result from traumatic injury and are characteristically sustained by relatively young adults who are otherwise in good health (15). In contrast, the majority of lower limb amputations are performed secondary to peripheral vascular disease (PVD: 16, 17, 18). The incidence of this condition increases with age, hence those typically undergoing PVD-related amputation are older than 60 years of age (19) and commonly experience concurrent medical conditions (17). Moreover, survival following major lower limb amputation for PVD is poor (20). 
The purpose of the study is to determine the prevalence of clinically significant affective distress (symptoms of depression and anxiety) in a sample of individuals with upper limb amputations and to examine the contribution of coping strategies to the prediction of psychosocial adjustment of individuals with acquired upper limb amputations. In the current research, psychosocial adjustment is conceptualized as the absence of clinically elevated symptoms of anxiety and depression and evidence of positive adjustment to amputation and prosthesis use.

\section{Methods}

\section{Study Design and Participants}

Eligible members of the British Limbless Ex-Service Men's Association (BLESMA), a British national charity dedicated to promotion of the welfare of those who have lost a limb or limbs, one or both eyes, or the use of a limb in any branch of Her Majesty's Forces or Auxiliary Forces were invited to participate in a research project concerning psychosocial adjustment to physical injury. Data were gathered using self-report postal questionnaires. An article outlining the aims of the study was published in BLESMA's quarterly magazine, the BLESMAG. Questionnaire packs including cover letters, a copy of the questionnaire and a stamped addressed reply envelope were subsequently distributed to eligible members. A reminder to return completed questionnaires to BLESMA head office was published in the BLESMAG approximately 6 weeks after initial questionnaire distribution. Personalised reminders were not issued to non-responders due to financial constraints and respondents were not offered incentives for participation. The Ethics Committee of the authors' institution approved the study protocol. 
Of the questionnaires distributed $(\mathrm{n}=2,500), 22$ were returned because the intended recipient was deceased. A total of 1,222 questionnaires were returned representing a response rate of $49 \%$, of which 1,072 contained sufficient data for analysis. Data were collected for 952 individuals with limb amputation(s). The inclusion criterion for the current investigation was acquired upper limb amputation. Analysis of the role of coping in adjustment to lower limb amputation has been detailed elsewhere (12). As eligible respondents $(n=141)$ were predominantly male $(n=139)$ and had injury $(n=$ 140) rather than disease-related amputations, 3 individuals ( 2 females and 1 male whose amputation was performed secondary to cancer) were excluded and analysis was limited to a sample of 138 males with amputations resulting from traumatic injury. Characteristics of the sample are outlined in Table 1. The mean age of respondents was 74.6 years $(\mathrm{SD}=11.4$; range 39 - 91; median 79 years). The average time elapsed since amputation was 50.2 years $(\mathrm{SD}=13.4$; range 5 - 63; median 57 years). Most amputations were at the above-elbow level (47.8\%). The majority of respondents reported using a prosthesis $(72.5 \%)$ although the average daily duration of prosthesis use varied considerably $(46 \%(n=46)$ used their prostheses for more than 8 hours per day; $19 \%$ reported daily prosthesis use of 2 to 8 hours; $35 \%$ reported occasional sporadic or task specific use e.g. driving). These rates and patterns of usage are broadly consistent with findings reported elsewhere $(2,21,22)$.

\section{Measures}

Coping strategies were assessed using the Coping Strategy Indicator (23). This thirtythree-item self-report questionnaire measures the use of three coping strategies, namely problem solving, seeking social support, and avoidance, in response to a specific stressor. Responses are indicated by means of a three point scale: a lot (3), a 
little (2), or not at all (1). The three subscales each contain 11 items and subscale scores are calculated by summing responses to appropriate items (range 0-33), higher scores indicate greater use of the strategy. Cronbach's alpha coefficients indicate adequate internal consistency for each of the subscales ranging from 0.86 to 0.98 for problem solving, 0.89 to 0.98 for seeking social support and from 0.77 to 0.96 for avoidance (23-27). In the original version of the scale respondents are asked to identify a stressful event occurring within the last six months and to consider the manner in which they had coped with it. In the current study 'coping with amputation' was specified as the event. Respondents were required to think of a problem encountered in relation to their injury in the past six months and to reflect on their coping strategies.

Symptoms of anxiety and depression were assessed using the Hospital Anxiety and Depression Scale (HADS: 28). This 14 item scale is intended as a brief screening instrument for both anxiety and depression in non-psychiatric populations (29). Items are answered on a four-point Likert scale (range 0 to 3 ). The anxiety and depression subscales each comprise 7 items that are summed to give subscale scores ranging from 0 to 21, with higher scores indicating greater levels of anxiety and depression. Good reliability has been reported for each of the HADS subscales (see 30 for review). Zigmond \& Snaith (28) recommend that, for the HADS anxiety and depression subscales alike, raw scores of between 8 and 10 identify 'mild' cases, 1114 'moderate' cases and 15 or greater 'severe' cases.

Psychosocial adaptation to prosthesis use was assessed using the psychosocial adjustment subscales of the Trinity Amputation and Prosthesis Experience Scales 
(TAPES: 31). Although this multidimensional assessment of adaptation to amputation and prosthesis use was originally developed with reference to lower limb amputation, the inherent objective of facilitating examination of the psychosocial processes involved in adjusting to an artificial limb, the specific demands of wearing a prosthesis and the potential sources of maladjustment (31), also has potential applicability and clinical relevance in cases of upper limb amputation (6) where such standardised assessments are lacking (32). Exploration of the factorial structure of the TAPES with individuals with acquired upper limb amputations has been reported elsewhere (6). In the current study the subscales assessing psychosocial adjustment to prosthesis use (i.e. general adjustment, social adjustment, adjustment to limitation and optimal adjustment) were utilised.

The optimal adjustment subscale incorporates two items reflecting the development of an optimistic outlook and the positive appraisal of life in spite of the trauma associated with amputation and the use of an artificial limb (6). The general adjustment subscale reflects the extent of adjustment to and acceptance of an artificial limb and incorporates items such as "As time goes by, I accept my artificial limb more" and "Although I have an artificial limb, my life is full". Social adjustment pertains to the influence of the artificial limb in social situations, encompassing ease of talking about the limb and dealing with others' reactions to it. The adjustment to limitation dimension reflects restriction ensuing from having an artificial limb and incorporates items such as "Having an artificial limb makes me more dependent on others than I would like" and "Being an amputee means that I can't do what I want to do". Items on each of these subscales are rated on 5-point Likert scales, with higher scores indicating more favourable adjustment. Cronbach's alpha coefficients indicate 
adequate internal reliability for each of the subscales (optimal adjustment .84, general adjustment .91, social adjustment .79, and adjustment to limitation .80) (6).

\section{Data Analyses}

Data were initially analyzed using descriptive statistics. Continuous variables are presented in terms mean value, standard deviation and range (see Table 2). Analyses based on TAPES subscales were confined to those currently using a prosthetic device $(n=100)$ as the questions pertain directly to aspects prosthesis use. There were no significant differences between prosthesis users and non-users in terms of endorsement of coping strategies, symptoms of anxiety or depression, hence the analyses of these variables are based on the group as a whole.

Intercorrelations among all variables were calculated to identify associations between hypothesized predictors and outcome measures (see Table 3 and Table 4). A series of standard multiple regressions were conducted to determine the extent to which coping strategies predicted adjustment. The independent variables in each regression equation were a set of three coping strategies, namely avoidance, seeking social support and problem solving. The predictor variables were entered in a single block. The dependent variables were participants' scores on the HADS subscales, and TAPES subscales. Standard beta weights were examined to determine the best unique predictors of adjustment (see table 5). Examination of tolerance/VIF values and condition indices indicated inconsequential collinearity. All calculations were computed with SPSS, Version 12.0.1.

\section{Results}




\section{Descriptive characteristics}

The means and standard deviations for all variables are presented in Table 2. HADSdepression scores ranged from 0 to 20 . The mean score was $5.42(\mathrm{SD}=4.18)$. Five individuals (3.6\%) had scores in the range representing 'severe' symptoms, while $7.2 \%$ of respondents $(\mathrm{n}=10)$ had scores in the range indicative of 'moderate' symptoms and $17.4 \%(\mathrm{n}=24)$ met the criterion for 'mild' symptoms of depression. Thus in total $28.3 \%$ met the criterion indicative of possible clinical depression. The average score on the HADS-anxiety subscale was 6.09 (SD 4.52). More than onethird $(35.5 \% ; \mathrm{n}=49)$ of participants met the criterion for possible clinical anxiety. Of these, $17.4 \%(n=24)$ scored within the mild range, $13.0 \%(n=18)$ reported moderate levels of anxiety and $5.1 \%(n=7)$ indicated severe anxiety levels.

\section{Relationship between demographic and outcome variables}

Neither anxiety nor depression, were correlated with age or time since amputation. Similarly, amongst prosthesis users none of the TAPES-psychosocial adjustment subscales were significantly correlated with age or time since amputation $(\mathrm{p}>.01)$.

\section{Correlations between predictor and outcome variables}

Table 3 illustrates the correlations between the predictor variables and depression and anxiety. Amongst the group of prosthesis users, avoidance was significantly associated with adjustment to limitation $(r=-.326)$, general adjustment $(r=-.330)$, optimal adjustment $(\mathrm{r}=-.486)$ and social adjustment $(\mathrm{r}=-.487)$. Associations between problem solving and seeking social support and the psychosocial adjustment to prosthesis use subscales were not significant (see table 4). 


\section{Regression analyses}

Table 5 provides a summary of the results obtained from the series of multiple regression analyses predicting the various facets of psychosocial adaptation to amputation.

When anxiety was used as an outcome measure $35.9 \%$ of the variance was explained by the block of coping strategies, $\mathrm{F}_{(3,112)}=20.88, \mathrm{p}<.001$. Examination of the beta weights revealed that greater use of avoidance was associated with higher levels of reported anxiety $(\beta=.604, \mathrm{t}=7.47, \mathrm{p}<.001)$. When predicting HADS-depression $32.8 \%$ of variance was explained by the block of coping strategies; $F_{(3,113)}=18.41, p$ $<.001$. Specifically, avoidance and problem solving made significant contributions to the prediction of depression scores. Higher levels of avoidance were associated with higher levels of reported depression, $(\beta=.611, t=7.39, p<.001)$ and greater use of problem solving was associated with lower levels of depressive symptomatology, $(\beta=$ $-.227, \mathrm{t}=-2.55, \mathrm{p}=.012)$.

In the prosthesis user group, $\left(\mathrm{F}_{(3,79)}=9.771, \mathrm{p}<.001\right), 27.1 \%$ of the variance in social adjustment was explained by the predictor variables. Examination of the beta weights indicates that avoidance was the strongest predictor of social adjustment ( $\beta=$ $-.555, \mathrm{t}=-5.367, \mathrm{p}<.001)$. When predicting general adjustment scores, $10.3 \%$ of the variance was explained $\left(\mathrm{F}_{(3,79)}=3.029, \mathrm{p}=.034\right)$ and when scores on the adjustment to limitation scale were entered as the dependent variable, $13.0 \%$ of the variance was explained; $\mathrm{F}_{(3,71)}=3.54, \mathrm{p}=.019$. In both instances, avoidance emerged as the strongest predictor $(\beta=-.334, \mathrm{t}=-2.91, \mathrm{p}=.005$; and $\beta=-.349, \mathrm{t}=-2.95, \mathrm{p}=.004$, respectively). The predictor variables explained $31.1 \%$ of the variance in optimal 
adjustment scores; $F_{(3,78)}=11.75, p<.001$. Two of the coping strategies, problem solving $(\beta=.321, \mathrm{t}=2.853, \mathrm{p}=.006)$ and avoidance $(\beta=-.567, \mathrm{t}=-5.646, \mathrm{p}<.001)$ contributed significantly to the variance explained.

\section{Discussion}

This study provides (1) prevalence data on symptoms of depression and anxiety in a sample of individuals with acquired upper limb amputations; and (2) the first reported investigation of the contribution of coping strategies to the prediction of psychosocial adjustment to upper limb amputation. The prevalence of depressive symptomatolgy (28.3\%) for the current sample was almost three times greater than rates reported in a non-clinical sample broadly representative of UK adults (33). This finding is consistent with the depression point prevalence estimate of $32 \%$, recently reported by Darnell et al. (34), in a U.S. sample of 100 community dwelling individuals with predominantly traumatic upper limb amputations. Prevalence of clinically significant anxiety symptoms (34.6\%) in the current sample is broadly consistent with general population rates (33). Comparable data for other upper limb amputation samples is not available, however, this result is consistent with findings premised on samples with lower limb amputations (e.g. 35). Previous research $(7,36)$ suggests recency of amputation as a significant risk factor for symptoms of anxiety and depression. These findings were not confirmed in the current analyses. Participants in the current research were at least 5 years post-amputation; it may be that the association between amputation recency and elevated risk for affective distress operates only in the early postoperative/rehabilitation period. 
In this study, psychosocial functioning of adults with acquired limb amputations was significantly related to coping strategies. Of the three coping strategies, avoidance was most consistently associated with poor psychosocial adaptation to amputation. This finding is consistent with previous research involving individuals with lower limb amputations $(7,12)$, and indeed other patient groups $(37,38)$, which suggests a significant association between avoidant coping strategies and higher levels of psychosocial and physical dysfunction. While some forms of avoidance, for example denial, may be beneficial in for short periods (39), if prolonged, avoidance may be detrimental because it prohibits individuals from engaging in more active coping efforts (40).

The association between problem solving and lower levels of depressive symptomatology and higher optimal adjustment scores are also in keeping with results of prior research based on lower limb amputation samples. For example, Livneh et al. (7) found that active problem solving influenced a range of psychosocial adjustment measures. In their analyses, higher levels of adjustment, acknowledgement, and acceptance of disability were associated with greater utilization of problem solving based strategies, as were lower levels of internalised anger and depressive symptomatology.

Although some previous studies have demonstrated significant associations between greater social support seeking and better functioning (41), higher quality of life (42), and lower rates of depressive symptomatology (12) in a variety of patient groups, consistent with most cross-sectional studies $(43,44)$ associations between seeking social support and psychosocial outcome measures were not evident in the current 
analyses. It may be that differences in situational characteristics such as stressor type, controllability, and duration (43), as well as the diversity of instruments used to measure social support seeking influenced these outcomes.

Some caution is warranted with respect to interpretation of these results. First, most members of BLESMA are mostly veterans with combat-related traumatic amputations, thus participants in the current research represent a specialised sample. The camaraderie and fellowship traditionally associated with military amputation may have led to bias in the findings. Furthermore, people who sustain traumatic limb amputation, whether military or civilian, are typically working-age adults in otherwise good health. The circumstances surrounding disease-related amputation, and the associated long-term prognoses, differ substantially from those surrounding traumatic amputation (45). Hence, the generalizeability of the current findings to cases of disease-related upper limb amputation requires investigation. Additionally, as the analyses reported here are based exclusively on male respondents the relevance of the findings to females' post-amputation experiences also requires verification. Second, the cross-sectional design of this study means that we cannot draw inferences about the causal link between coping and adjustment variables. Moreover, cross-sectional studies of this nature are not suitable for examination of longitudinal fluctuations in coping styles. A longitudinal design would facilitate exploration of process-oriented coping activities which are necessarily dynamically interacting and evolving. Indeed, it's likely that findings emerging from this cross-sectional study of individual's with predominantly long-term amputations may differ from those found in samples at shorter follow-up times as previous research suggests that that psychosocial adaptation to amputation occurs over time $(7,46)$. Third, although a substantial 
number of respondents met the criteria for clinically significant depression, the HADS utilized to measure this construct is not intended as substantiation of diagnosis, rather it serves as a screening measure. Fourth, the survey response rate and self-report nature of the design may have resulted in bias in the analysis. Much of the questionnaire pertained to prosthesis use, thus non-prosthesis users may not have been motivated to take part potentially leading to over-representation of well-adjusted, highly functioning prosthesis users in the sample. Furthermore, self-reports may be affected by respondents' self-awareness or cognitive functioning. Nonetheless, assessment of psychological status is premised on the notion that in most cases only the individual can provide meaningful data about their psychological state. In addition, in common with all mail surveys, the conditions under which the questionnaires were completed were uncontrolled and may have affected the findings.

With these limitations in mind, the favourable associations between problem solving and psychosocial outcomes, and the maladaptive consequences of the use of avoidant coping strategies evidenced here suggests the potential benefits of interventions to reduce reliance on avoidant coping and stimulate more problem-focussed approaches to coping with difficulties and challenges in order to facilitate adaptation and prevent problems in psychosocial functioning. This study also highlights the elevated prevalence of depressive symptomatology in this upper limb amputation group and suggests the need for a comprehensive follow-up services providing specialist physical and psychological support where necessary. 


\section{References}

1. Gaine WJ, Smart C, Bransby-Zachary M. Upper limb traumatic amputees. Review of prosthetic use. J Hand Surg [Br] 1997;22:73-76.

2. Dudkiewicz I, Gabrielov R, Seiv-Ner I, Zelig G, Heim M. Evaluation of prosthetic usage in upper limb amputees. Disabil Rehabil 2004;26:60-63.

3. Kooijman CM, Dijkstra PU, Geertzen JHB, Elzinga A, van der Schans CP. Phantom pain and phantom sensations in upper limb amputees: an epidemiological study. Pain 2000;87:33-41.

4. Pillet J, Didierjean-Pillet A. Aesthetic hand prosthesis: gadget or therapy? Presentation of a new classification. J Hand Surg [Br] 2001;26:523-528.

5. Esquenazi A. Amputation rehabilitation and prosthetic restoration. From surgery to community reintegration. Disabil Rehabil 2004;26:831-836.

6. Desmond DM, MacLachlan M. The factor structure of the Trinity Amputation and Prosthesis Experience Scales (TAPES) with individuals with acquired upper limb amputations. Am J Phys Med Rehabil 2005;84:506-513.

7. Livneh H, Antonak RF, Gerhardt J. Psychosocial adaptation to amputation: the role of sociodemographic variables, disability related factors and coping strategies. Int J Rehabil Res 1999;22:21-31.

8. Endler NS, Corace KM, Summerfeldt LJ, Johnson JM, Rothbart P. Coping with chronic pain. Pers Individ Dif 2003;34:323-346.

9. Livneh H, Antonak RF, Gerhardt J. Multidimensional investigation of the structure of coping among people with amputations. Psychosomatics 2000;41:235244. 
10. Mikulinecer M, Florian V. Coping and adaptation to trauma and loss. In:

Zeidner M, Endler NS, editors. Handbook of Coping: Theory, Research and Applications. New York: John Wiley and Sons Inc.; 1996. p. 554-572.

11. Dunn D. Well-being following amputation: salutary effects of positive meaning, optimism and control. Rehabil Psychol 1996;41:245-302.

12. Desmond DM, MacLachlan M. Coping strategies as predictors of psychosocial adaptation in a sample of elderly veterans with acquired lower limb amputations. Soc Sci Med 2006;62:208-216.

13. Hill A, Niven CA, Knussen C. The role of coping in adjustment to phantom limb pain. Pain 1995;62:79-86.

14. Horgan O, MacLachlan M. Psychosocial adjustment to lower-limb amputation: A review. Disabil Rehabil 2004;26:837-850.

15. National Amputee Statistical Database. The Amputee Statistical Database for the UK Annual Report. Edinburgh; 2003/04.

16. Helm P, Engel T, Holm A, Kristiansen VB, Rosendahl S. Function after lower limb amputation. Acta Orthop Scand 1986;57:154-157.

17. Stewart CPU, Jain AS. Cause of death of lower limb amputees. Prosthet Orthot Int 1992;16:129-132.

18. Pernot HF, de Witte LP, Lindeman E, Cluitmans J. Daily functioning of the lower extremity amputee: an overview of the literature. Clin Rehabil 1997;11:93-106. 19. Ebskov LB. Lower limb amputations for vascular insufficiency. Int J Rehabil Res 1991;14:59-64.

20. Pell JP, Stonebridge P. Association Between Age and Survival Following Major Amputation. Eur J Vasc Endovasc Surg 1999;17:166-169. 
21. Datta D, Selvarajah K, Davey N. Functional outcome of patients with proximal upper limb deficiency acquired and congenital. Clin Rehabil 2004;18:172177.

22. Roeschlein RA, Domholdt E. Factors related to successful upper extremity prosthetic use. Prosthet Orthot Int 1989;13:14-18.

23. Amirkhan JH. A factor analytically derived measure of coping: the Coping Strategy Indicator. J Pers Soc Psychol 1990;59:1066-1074.

24. Utsey SO, Ponterotto JG, Reynolds AL, Cancelli AA. Racism and discrimination, coping, life satisfaction, and self-esteem among African Americans. J Couns Dev 2000;78:72-80.

25. Bijttebier P, Vertommen H. Psychometric properties of the Coping Strategy Indicator in a Flemish sample. Pers Individ Dif 1997;23:157-160.

26. Clark KK, Bormann CA, Cropanzano RA, James K. Validation evidence for three measures of coping. J Pers Assess 1995;65:434-455.

27. Desmond DM, Shevlin M, MacLachlan M. Dimensional analysis of the coping strategy indicator in a sample of elderly veterans with acquired limb amputations. Pers Individ Dif 2006;40:249-259.

28. Zigmond AS, Snaith RP. The Hospital Anxiety and Depression Scale. Acta Psychiatr Scand 1983;67:361-370.

29. Herrmann C. International experiences with the Hospital Anxiety and Depression Scale- a review of validation data and clinical results. J Psychosom Res $1997 ; 42: 17-41$.

30. Bjelland I, Dahl AA, Haug TT, Neckelmann D. The validity of the Hospital Anxiety and Depression Scale: An updated literature review. J Psychosom Res 2002;52:69-77. 
31. Gallagher P, MacLachlan M. Development and psychometric evaluation of the Trinity Amputation and Prosthesis Experience Scales (TAPES). Rehabil Psychol 2000;45:130-154.

32. Davidson J. A comparison of upper limb amputees and patients with upper limb injuries using the Disability of the Arm, Shoulder and Hand (DASH). Disabil Rehabil 2004;24:917-923.

33. Crawford JR, Henry JD, Crombie C, Taylor EP. Normative data for the HADS from a large non-clinical sample. Br J Clin Psychol 2001;40:429-434.

34. Darnall BD, Ephraim P, Wegener ST, Dillingham T, Pezzin L, Rossbach P, et al. Depressive symptoms and mental health service utilization among persons with limb loss: Results of a national survey. Arch Phys Med Rehabil 2005;86:650-658. 35. Bodenheimer C, Kerrigan AJ, Garber SL, Monga TN. Sexuality in persons with lower extremity amputations. Disabil Rehabil 2000;22:409-415.

36. Kashani JH, Frank RG, Kashani SR, Wonderlich SA, Reid JC. Depression among amputees. J Clin Psychiatry 1983;44:256-258.

37. Spangenberg JJ, Theron JC. Stress and coping strategies in spouses of depressed patients. J Psychol 1999;133:253-262.

38. Welch JL, Austin JK. Stressors, coping and depression in haemodialysis patients. J Adv Nurs 2001;33:200-207.

39. Levine J, Warrenburg S, Kerns R, Schwartz G, Delaney R, Fontana A, et al. The role of denial in recovery from coronary heart disease. Psychosom Med 1987;49:109-117.

40. Suls J, Fletcher B. The relative efficacy of avoidant and nonavoidant coping strategies: a meta-analysis. Health Psychol 1985;4:249-88. 
41. Scharloo M, Kaptein AA, Weinman J, Hazes JM, Willems LNA, Bergman W, et al. Illness perceptions, coping and functioning in patients with rheumatoid arthritis, chronic obstructive pulmonary disease and psoriasis. J Psychosom Res 1998;44:573585.

42. Hopman-Rock M, Kraaimaat FW, Bijlsma JWJ. Quality of life in elderly subjects with pain in the hip or knee. Qual Life Res 1997;6:67-76.

43. Penley JA, Tomaka J, Wiebe JS. The Association of Coping to Physical and Psychological Health Outcomes: A Meta-Analytic Review. J Behav Med 2002;25:551-603.

44. Schreurs KMG, de Ridder DTD. Integration of coping and social support perspectives: Implications for the study of adaptation to chronic diseases. Clin Psychol Rev 1997;17:89-112.

45. Dougherty PJ. Transtibial amputees from the Vietnam War. Twenty-eight-year follow-up. J Bone Joint Surg Am 2001;83-A:383-389.

46. Gallagher P, MacLachlan M. Adjustment to an artificial limb: a qualitative perspective. J Health Psychol 2001;6:85-100. 